PSFC/JA-08-28

Parametric Analysis of the

Two-Fluid Tearing Instability

E. Ahedo ${ }^{\dagger}$ and J.J. Ramos

November, 2008

Plasma Science and Fusion Center

Massachusetts Institute of Technology

Cambridge MA 02139, U.S.A.

† Permanent address: Universidad Politécnica de Madrid. Escuela Técnica Superior de Ingenieros Aeronáuticos. 28040 Madrid, Spain.

This work was supported by the Ministerio de Ciencia e Innovación of Spain under Project ESP200762694 and by the U.S. Department of Energy under Grants Nos. DEFG02-91ER54109 and DEFC0208ER54969. Reproduction, translation, publication use and disposal, in whole or in part, by or for the United States government is permitted. 


\title{
Parametric analysis of the two-fluid tearing instability
}

\author{
Eduardo Ahedo * and Jesús J. Ramos \\ Plasma Science and Fusion Center, Massachusetts Institute of Technology, Cambridge, MA, USA
}

November 12, 2008

\begin{abstract}
A two-fluid analysis of the current driven tearing instability is presented. It concentrates on the systematic investigation of the physics related to the contributions of the Hall term and the electron pressure gradient to the electric field, for arbitrary values of the ion skin depth and of the magnitude of the magnetic guide field. The plasma compressibility is treated consistently for a wide range of the plasma beta that excludes only the extremely cold limit where the mode growth rate would become sonic or supersonic. Conversely, the effects associated with the finite ion gyroradius and the equilibrium density and temperature gradients are neglected. Seven parametric regions are identified, characterized by the relative strengths of the Hall and beta parameters. Five of them are amenable to asymptotic analyses yielding analytic dispersion relations and one allows a semi-analytic treatment. The singular, multi-layer structure of the tearing mode and the conditions under which the different components of the magnetic field diffuse are shown in detail for each of those parametric regions.
\end{abstract}

PACS numbers: 52.30.Ex, 52.35.Py

\footnotetext{
*Permanent address: Universidad Politécnica de Madrid, Escuela Técnica Superior de Ingenieros Aeronáuticos, Plaza Cardenal Cisneros, 28040 Madrid; eduardo.ahedo@upm.es
} 


\section{Introduction}

The extension of the classic resistive-MHD tearing mode theory [1] to a two-fluid plasma model incorporates a variety of new physics and has been the subject of numerous studies (see, e.g. the detailed discussion and bibliography in Ref.[2]). More recently, there has been a renewed interest in accurate two-fluid analytic results that can be used for verification of the new extended-MHD simulation codes. In this regard, the work of Ref.[2] provides a broad and updated analysis of the linear tearing instability based on the so called Hall-MHD model, which is the simplest extension of single-fluid MHD, accounting for distinct ion and electron flows and bringing in physical effects at the length scales of the ion skin depth and the ion sound gyroradius, while still neglecting the diamagnetic effects associated with a finite ion Larmor radius. Despite its rather general scope, the analysis of Ref.[2] has some limitations, even within the physical model and the specific instability under consideration. In particular, it considers only the "large magnetic aspect ratio" or "strong magnetic guide field" limit, where the equilibrium magnetic field has a large component perpendicular to the reconnection plane, relative to its component on that plane. More notably, the ordering schemes leading to the newly proposed asymptotic dispersion relations become inaccurate as the ion skin depth becomes smaller than the macroscopic equilibrium length scale and fail to recover the classic single-fluid result in the limit of vanishing ion skin depth. The purpose of the present work is to put forward a complementary study of the linear two-fluid tearing instability that will be continuously accurate for ion skin depths ranging from zero to the equilibrium length scale, based on the same Hall-MHD plasma model and a similar one-dimensional slab equilibrium without density or temperature gradients, but with arbitrary magnetic aspect ratio. Unlike Ref.[2] that extends the analysis to explore the limits of extremely low or zero beta (where the tearing growth rate becomes sonic or supersonic) and very large values of the dimensionless tearing instability index $\Delta^{\prime} / k$, we will restrict ourselves to consideration of $\Delta^{\prime} / k \lesssim 1$ and to at least a minimal value of beta that guarantees subsonic growth rates. These conditions are well satisfied in most applications of interest to magnetically confined plasmas and, in return for these mild restrictions, we will be able to develop a unified formulation that covers general ion skin depths, arbitrary magnetic aspect ratios and the subsonic beta range of main interest, including the complete account of 
plasma compressibility.

With our working hipotheses of subsonic growth and $\Delta^{\prime} / k \lesssim 1$, we will obtain a scaled growth rate that depends on only two independent combinations of primary input data, as the eigenvalue of a compact, unified Hall-MHD tearing mode system, Eqs.(52),(53),(86),(87), valid for the whole relevant parameter space. The two independent input combinations are appropriately scaled versions of the plasma beta, defined here as $\beta=c_{s}^{2} / c_{A}^{2}$ where $c_{s}$ and $c_{A}$ are the sound and Alfven velocities respectively, and the Hall parameter $\alpha=k d_{i}$ where $d_{i}$ is the ion skin depth and $2 \pi / k$ is the mode wavelength along its propagating direction. Three well known dispersion relations will be recovered in three asymptotic domains of our two-dimensional parameter space: for sufficiently small values of the Hall parameter we will get the single-fluid tearing dispersion relation [1], for sufficiently large values of both beta and the Hall parameter we will get the dispersion relation derived with the so-called "electron-MHD" model $[3,4]$ and for sufficiently small beta and large Hall parameter we will get the so-called "semicollisional" tearing dispersion relation $[5,6,7]$. We will also carry out the detailed asymptotic analysis of the three parametric regions that cover the transitions between these three classic limits. For sufficiently high beta and general values of the Hall parameter, we will derive a novel analytic dispersion relation that connects the "single-fluid" and "electron-MHD" forms. For sufficiently large values of the Hall parameter and general betas, a case whose large magnetic aspect ratio limit was covered by the analysis of Ref.[2], we will find the same analytic dispersion relation that connects the "electron-MHD" and "semicollisional" forms, now applicable to arbitrary aspect ratios. As far as we are aware, there are no previous detailed studies of the transitional regime between the "single-fluid" and "semicollisional" dispersion relations at sufficiently low beta and our work will provide a new semi-analytic treatment of this regime.

The paper is organized as follows. Section 2 presents the Hall-MHD model to be considered, with its slab equilibrium and general first-order linearized equations, and identifies the dimensionless parameters of the problem. Section 3 carries out the multiple spatial scale reduction for small resistivity and derives our basic tearing mode system for the non-ideal inner layer, written in terms 
of appropriately scaled variables. Section 4 is devoted to the solution of the inner layer equations in the different asymptotic parameter regimes. For each of these, the small-scale structure of the tearing mode and the conditions under which the different components of the magnetic field diffuse are discussed in detail. Section 5 gives the general dispersion relation and its various limits in the different parametric regions. A summarizing discussion is presented in Section 6. The main body of the paper is written based on a massless electron model, although the extension to include the effect of a small but finite electron mass on the linear tearing mode is straightforward. The generalization to a finite electron inertia and the modified asymptotic dispersion relations in the collisionless limit, when the inertial term dominates over the resistive friction force in the electron momentum conservation equation, are dealt with in an Appendix.

\section{The model}

The basic plasma description to be adopted in this work is provided by a two-fluid, Hall-MHD model with massless electrons and zero-Larmor-radius ions, closed with polytropic equations of state and with an Ohmic resistive term in the generalized Ohm's law as its sole diffusivity:

$$
\begin{aligned}
& \frac{\partial \boldsymbol{B}}{\partial t}=-\nabla \times \boldsymbol{E}, \\
& \nabla \cdot \boldsymbol{B}=0 \\
& \mu_{0} \boldsymbol{j}=\nabla \times \boldsymbol{B}, \\
& \frac{\partial \rho}{\partial t}+\nabla \cdot \rho \boldsymbol{v}=0 \\
& \rho \frac{D \boldsymbol{v}}{D t}=\boldsymbol{j} \times \boldsymbol{B}-\nabla p=\frac{1}{\mu_{0}}(\boldsymbol{B} \cdot \nabla) \boldsymbol{B}-\nabla W, \\
& \boldsymbol{E}=-\boldsymbol{v} \times \boldsymbol{B}+\eta \boldsymbol{j}+\frac{1}{e n}\left(\boldsymbol{j} \times \boldsymbol{B}-\nabla p_{e}\right)=-\boldsymbol{v} \times \boldsymbol{B}+\eta \boldsymbol{j}+\frac{m_{i}}{e}\left(\frac{D \boldsymbol{v}}{D t}+\frac{\nabla p_{i}}{\rho}\right), \\
& p_{s} n^{-\Gamma_{s}}=\text { const } \quad(s=i, e),
\end{aligned}
$$

where $n$ and $\rho=m_{i} n$ are the particle and mass densities respectively, $p=p_{i}+p_{e}$ and the sum of kinetic and magnetic pressures $W=p+B^{2} / 2 \mu_{0}$ will be used preferentially instead of $p$; the resistivity $\eta$ will be taken as a constant and the rest of symbols are conventional. 
As zeroth-order solution we will assume a stationary, force-free equilibrium with constant density and temperatures:

$$
\rho_{0}, p_{s 0}=\mathrm{const}, \quad \boldsymbol{j}_{0} \times \boldsymbol{B}_{0}=\mathbf{0}, \quad \boldsymbol{v}_{0}=\mathbf{0}, \quad \boldsymbol{E}_{0}=\eta \boldsymbol{j}_{0} \simeq \mathbf{0},
$$

where the last condition holds for times smaller than the resistive diffusion time, assumed to be very long. A one-dimensional equilibrium slab geometry will also be assumed, with the inhomogeneity along the $x$ direction and the magnetic field of the form $\boldsymbol{B}_{0}=B_{0 y}(x) \boldsymbol{e}_{y}+B_{0 z}(x) \boldsymbol{e}_{z}$, so that the force-free condition requires that its magnitude $B_{0}$ be constant. Specifically, we will consider the sheet pinch profiles:

$$
B_{0 y}(x)=\epsilon_{B} B_{0} \tanh \frac{x}{L} \quad \text { and } \quad B_{0 z}(x)=\left[B_{0}^{2}-B_{0 y}^{2}(x)\right]^{1 / 2} .
$$

The constant $\epsilon_{B}$ is a measure of the relative strength of the $B_{0 y}$ magnetic component and is taken as much less than unity in the customary "strong magnetic guide field" approximation [2]. In the present work, however, we will consider arbitrary guide fields and will treat $\epsilon_{B}$ as a parameter of order unity. The components of the electric current density are

$$
j_{0 z}=\frac{1}{\mu_{0}} \frac{d B_{0 y}}{d x}=\frac{\epsilon_{B} B_{0}}{\mu_{0} L}\left(1-\tanh ^{2} \frac{x}{L}\right) \quad \text { and } \quad j_{0 y}=-\frac{1}{\mu_{0}} \frac{d B_{0 z}}{d x}=j_{0 z} \frac{B_{0 y}}{B_{0 z}},
$$

thus $L$ characterizes the width of the current sheet.

The first-order equations, obtained by linearizing the basic system (1-7) about our zeroth-order equilibrium, are:

$$
\begin{aligned}
& \frac{\partial \boldsymbol{B}_{1}}{\partial t}=-\nabla \times \boldsymbol{E}_{1}, \\
& \nabla \cdot \boldsymbol{B}_{1}=0, \\
& \mu_{0} \boldsymbol{j}_{1}=\nabla \times \boldsymbol{B}_{1}, \\
& \boldsymbol{E}_{1}=-\boldsymbol{v}_{1} \times \boldsymbol{B}_{0}+\eta \boldsymbol{j}_{1}+\frac{m_{i}}{e}\left(\frac{\partial \boldsymbol{v}_{1}}{\partial t}+\frac{\nabla p_{i 1}}{\rho_{0}}\right),
\end{aligned}
$$




$$
\begin{aligned}
& \frac{\partial \rho_{1}}{\partial t}+\rho_{0} \nabla \cdot \boldsymbol{v}_{1}=0, \\
& \mu_{0} \rho_{0} \frac{\partial \boldsymbol{v}_{1}}{\partial t}=\left(\boldsymbol{B}_{1} \cdot \nabla\right) \boldsymbol{B}_{0}+\left(\boldsymbol{B}_{0} \cdot \nabla\right) \boldsymbol{B}_{1}-\mu_{0} \nabla W_{1}, \\
& p_{1}=c_{s}^{2} \rho_{1}=W_{1}-\boldsymbol{B}_{0} \cdot \boldsymbol{B}_{1} / \mu_{0},
\end{aligned}
$$

where $c_{s}^{2}=\left(\Gamma_{e} T_{e 0}+\Gamma_{i} T_{i 0}\right) / m_{i}$ is the square of the sound velocity. Since $\rho_{0}$ is constant, the first-order ion pressure gradient can be absorbed with a redefinition of the electric field:

$$
\boldsymbol{E}_{1}-\frac{m_{i}}{e \rho_{0}} \nabla p_{i 1} \rightarrow \boldsymbol{E}_{1}
$$

For our present purposes of studying the linear tearing instability as a singular perturbation problem with small resistivity, the generalization of the above system to allow for a small but finite electron inertia is straightforward and amounts simply to a redefinition of the resistivity $\eta$. The details are given in the Appendix.

Normal mode perturbations independent of $z$, with periodic spatial variation along the $y$ direction and growth rate $\gamma$ will be considered,

$$
f(x, y, t)-f_{0}(x)=f_{1}(x) \exp (\gamma t+i k y),
$$

so that the wavenumber vector satisfies $\boldsymbol{k} \cdot \boldsymbol{B}_{0}=0$ at the singular surface $x=0$.

The set (11)-(17) consists of 14 scalar equations that can be split into a group of 6 first-order differential equations for $E_{1 y}, \boldsymbol{B}_{1}, W$, and $v_{1 x}$, and a group of 8 equations that yield algebraically $E_{1 x}, E_{1 z}, \boldsymbol{j}_{1}, v_{1 y}, v_{1 z}$ and $\rho_{1}$ in terms of the other six scalar variables. Once these 8 variables are substituted, the six differential equations are:

$$
\begin{aligned}
& i B_{1 x}^{\prime}=k B_{1 y} \\
& \begin{aligned}
\gamma \mu_{0} \rho_{0} E_{1 y}^{\prime} & =-\left(\gamma^{2} \mu_{0} \rho_{0}+k^{2} B_{0 y}^{2}+\eta \gamma \rho_{0} k^{2}\right) B_{1 z} \\
& \quad-k^{2} B_{0 z}\left(\mu_{0} W_{1}-B_{0 y} B_{1 y}\right)-k \frac{B_{0 y}^{\prime} B_{0}^{2}}{B_{0 z}^{2}} i B_{1 x}+k \frac{i m_{i}}{e} \gamma^{3} \mu_{0} \rho_{0} \xi, \\
\eta B_{1 z}^{\prime}= & \mu_{0}\left(B_{0 z} \gamma \xi-E_{1 y}\right)-\frac{i m_{i}}{e \rho_{0}}\left[B_{0 y}^{\prime} i B_{1 x}+k\left(\mu_{0} W_{1}-B_{0 y} B_{1 y}\right)\right],
\end{aligned}
\end{aligned}
$$




$$
\begin{aligned}
& \eta k B_{1 y}^{\prime}=\left(\mu_{0} \gamma+\eta k^{2}\right) i B_{1 x}+\mu_{0} k B_{0 y} \gamma \xi-\frac{i m_{i}}{e \rho_{0}} k B_{0 y}\left(\frac{B_{0 y}^{\prime}}{B_{0 z}} i B_{1 x}+k B_{1 z}\right) \\
& \gamma^{2} \mu_{0} \rho_{0} \xi^{\prime}=-k B_{0 y}^{\prime} i B_{1 x}-k^{2}\left(\mu_{0} W_{1}-B_{0 y} B_{1 y}\right)-\gamma^{2} \mu_{0} \rho_{1} \\
& \mu_{0} W_{1}^{\prime}=k B_{0 y} i B_{1 x}-\gamma^{2} \mu_{0} \rho_{0} \xi
\end{aligned}
$$

where the prime $\left({ }^{\prime}\right)$ denotes the derivative with respect to $x$; we have introduced the Lagrangian displacement variable

$$
\xi=\gamma^{-1} v_{1 x}
$$

and the perturbed density, satisfying the algebraic relation

$$
\left(c_{s}^{2} k^{2}+\gamma^{2}\right) \rho_{1}=-\gamma^{2} \rho_{0} \xi^{\prime}-\frac{k B_{0 z}}{\mu_{0}}\left(k B_{1 z}+\frac{B_{0 y}^{\prime}}{B_{0 z}} i B_{1 x}\right),
$$

is to be substituted in Eq. (23).

This set of first-order equations can be written, in a more conventional way, as the following set of 3 second-order differential equations for $\left(\xi, B_{1 x}, B_{1 z}\right)$ :

$$
\begin{aligned}
& \gamma^{2} \mu_{0}\left(\rho_{0} \nabla^{2} \xi+\rho_{1}^{\prime}\right)=i k\left(B_{0 y} \nabla^{2} B_{1 x}-B_{0 y}^{\prime \prime} B_{1 x}\right) \\
& \eta \nabla^{2} B_{1 x}=\gamma \mu_{0}\left(B_{1 x}-i k B_{0 y} \xi\right)-\frac{m_{i}}{e \rho_{0}} k B_{0 y}\left(k B_{1 z}+\frac{B_{0 y}^{\prime}}{B_{0 z}} i B_{1 x}\right) \\
& \eta \gamma \rho_{0} \nabla^{2} B_{1 z}=k B_{0 y}^{2}\left(k B_{1 z}+\frac{B_{0 y}^{\prime}}{B_{0 z}} i B_{1 x}\right)-\gamma^{2} \mu_{0}\left(B_{0 z} \rho_{1}-\rho_{0} B_{1 z}\right)-\frac{m_{i}}{e} \gamma\left(B_{0 y} \nabla^{2} B_{1 x}-B_{0 y}^{\prime \prime} B_{1 x}\right),
\end{aligned}
$$

with $\nabla^{2} \equiv d^{2} / d x^{2}-k^{2}$ and the algebraic equation (27) for $\rho_{1}$. It is worth noting that Eqs. (25)-(28) follow exactly from the general linearized set, Eqs. (11)-(17), wihout any simplifying assumptions. In the limit $\epsilon_{B} \ll 1$, these equations recover the model considered by Mirnov et al.[2], except that they use a second-order differential equation for $\rho_{1}$ instead of our algebraic equation (25). 
The linear stability analysis must determine the dimensionless growth rate

$$
\epsilon_{\gamma}=\frac{\gamma}{c_{A} k}
$$

in terms of the five independent dimensionless parameters of the problem:

$$
\epsilon_{\eta}=\frac{\eta k}{\mu_{0} c_{A}}, \quad \epsilon_{B}=\frac{B_{0 y}(\infty)}{B_{0}}, \quad \alpha=k d_{i}, \quad \beta=\frac{c_{s}^{2}}{c_{A}^{2}} \quad \text { and } \quad k L
$$

where $c_{A}=B_{0}\left(\mu_{0} \rho_{0}\right)^{-1 / 2}$ is the Alfven velocity and $d_{i}=m_{i}\left(e^{2} \mu_{0} \rho_{0}\right)^{-1 / 2}$ is the ion skin depth. The parameter $\epsilon_{\eta}$ is the inverse of the magnetic Reynolds number $S$ based on the length $k^{-1}$. In addition to the ion skin depth characteristic of the Hall effects, one can define the lengths $d_{s}=d_{i} \sqrt{\beta}=m_{i} c_{s} /\left(e B_{0}\right), d_{\eta}=\epsilon_{\eta} / k$, and $d_{\gamma}=\epsilon_{\gamma} / k$, associated with compressible, resistive, and inertial effects, respectively. Table 1 details typical values of these parameters in some tokamak plasmas. Notice the extremely low value of $\epsilon_{\eta}$, which will be our fundamental expansion parameter. Resistive effects will matter only within a thin layer around $x=0$ where $\boldsymbol{k} \cdot \boldsymbol{B}_{0} \simeq 0$ [1], leading to the familiar, multiple scale tearing mode analysis for $\epsilon_{\eta} \rightarrow 0$. As mentioned before, the magnetic geometry inverse aspect ratio parameter $\epsilon_{B}$ will be assumed to be of order unity, thus allowing for arbitrary magnetic guide fields. Similarly, the Hall parameter $\alpha$ will only be restricted to satisfy $\alpha \lesssim \epsilon_{\eta}^{-1 / 5}$, thus covering all the practical range from the single-fluid to the strong-Hall regimes. Our only constraint on the beta parameter will be $\beta \gg \epsilon_{\gamma}^{2}$, which is well satisfied in tokamak plasmas and excludes just the extremely cold limit where the tearing mode growth rate would become sonic or supersonic. Finally, $k L$ will be taken as order unity but required to be such that the tearing instability index $k^{-1} \Delta^{\prime}$ is also of order unity or less: the exclusion of very large values of $k^{-1} \Delta^{\prime}$, which is sensible for most realistic applications, turns out in fact to be the most restrictive condition in our analysis. 


\section{Multiple spatial scale formulation}

The linear tearing mode analysis is based on the classic singular perturbation theory for $\epsilon_{\eta} \ll 1$ [1], that yields a growth rate scaling as a fractional power of the resistivity, $\epsilon_{\gamma} \propto \epsilon_{\eta}^{\nu}(0<\nu<1)$, and a mode eigenfunction structure on multiple spatial scales, resistive effects being important only within a microscopic region near $x=0$ where $\boldsymbol{k} \cdot \boldsymbol{B}_{0} \simeq 0$.

In the outer region where $k x$ and $x / L$ are considered to be of order unity, one can take the dissipationless and quasi-stationary limits, $\epsilon_{\eta} \rightarrow 0$ and $\epsilon_{\gamma} \rightarrow 0$. Then, in their leading orders, Eq.(26) reduces to

$$
B_{0 y} B_{1 x}^{\prime \prime}-\left(k^{2} B_{0 y}+B_{0 y}^{\prime \prime}\right) B_{1 x}=0
$$

and Eq.(28) reduces to

$$
k B_{1 z}=-i \frac{B_{0 y}^{\prime}}{B_{0 z}} B_{1 x}
$$

Moreover, combining Eq.(26) and Eq.(28) and anticipating the scaling $\epsilon_{\gamma} \propto \epsilon_{\eta}^{\nu} \gg \epsilon_{\eta}$, one finds that $k B_{1 z}+i B_{0 y}^{\prime} B_{0 z} / B_{1 x}$ will scale proportional to $\epsilon_{\gamma}^{2}$, hence the leading order of Eq.(27) yields

$$
k \xi=-i B_{1 x} / B_{0 y}
$$

and the leading order of Eq.(25) yields

$$
\rho_{1}=0 .
$$

Therefore, this outer region system, Eq.(31)-Eq.(34), is identical to the one obtained in ideal-MHD but is valid for any value of $\alpha$ and, like ideal-MHD at marginal stability, is incompressible for any value of $\beta$.

For the sheet pinch equilibrium profile of Eq. (9), the solution to Eq. (31) satisfying the boundary 
conditions $B_{1 x}( \pm \infty)=0$ is

$$
B_{1 x}(x)=B_{1 x}(0) \mathrm{e}^{-k|x|}\left(1+\frac{1}{k L} \tanh \frac{|x|}{L}\right)
$$

and Figure 1 illustrates the corresponding three components of the perturbed magnetic field. There is a discontinuity in $B_{1 x}^{\prime}=-i k B_{1 y}$ and $B_{1 z}^{\prime}$ at $x=0$,

$$
\frac{\left.B_{1 x}^{\prime}\right|_{x=0^{-}} ^{x=0^{+}}}{B_{1 x}(0)}=\frac{-\left.i k B_{1 y}\right|_{x=0^{-}} ^{x=0^{+}}}{B_{1 x}(0)}=\frac{\left.B_{1 z}^{\prime}\right|_{x=0^{-}} ^{x=0^{+}}}{B_{1 z}(0)}=\Delta^{\prime},
$$

with

$$
k^{-1} \Delta^{\prime}=2\left[(k L)^{-2}-1\right] .
$$

The condition for tearing mode instability is $\Delta^{\prime}>0[1]$, corresponding to the range $k L<1$. Notice that, for finite magnetic aspect ratios, the component $B_{1 z}$ is comparable to $B_{1 x}$ and should not be neglected: $B_{1 z}(0) / B_{1 x}(0)=\epsilon_{B} /(k L)$.

For $x \ll L$, one has

$$
B_{0 y} \simeq \epsilon_{B} B_{0} x / L \text { and } \quad B_{0 z} \simeq B_{0}
$$

so that Eq. (33) implies that $i k \xi \propto L / x \rightarrow \infty$ for $x / L \rightarrow 0$. A boundary layer must develop around $x=0$ in order to bound $k \xi$ and smooth the discontinuities in the components of the perturbed magnetic field, matching regularly the $x<0$ and $x>0$ branches of the outer solution. This boundary layer, where non-ideal effects neglected in the outer region must be taken into account, must have a width much smaller than the equilibrium current sheet width, $L$, and may include several distinct asymptotic sublayers depending on the plasma parameters. Within it, equilibrium quantities can be approximated by the leading terms of their Taylor expansions about $x=0$, Eq. (38), and the length scales associated with the gradients of perturbed quantities along $x$ are much shorter than $L$. In particular, the Laplacian operator acting on perturbed quantities can be approximated as $\nabla^{2} \simeq d^{2} / d x^{2}$. The only exception is $B_{1 x}$ (whose outer solution is continuous) that varies on the scale of $L$, although its discontinuous first derivative $d B_{1 x} / d x=-i k B_{1 y}$ varies on the 
short scales of the boundary layer. Accordingly, we will adopt the "constant- $B_{1 x}$ approximation" whereby $B_{1 x}$ is replaced by the constant $B_{1 x}(0)$ through the non-ideal layer, while its derivative is still treated as an $x$-dependent dynamical variable. The $B_{1 z}$ component has a continuous outer solution, Eq. (32), and might therefore have been expected to exhibit the same slower variation as $B_{1 x}$. This is indeed the case in single-fluid resistive-MHD, but the two-fluid Hall effects give rise to an additional, fast-varying and internally localized, contribution to $B_{1 z}$ with the opposite (odd) parity. This two-fluid, odd part of $B_{1 z}$ is conveniently represented by the variable

$$
Q=B_{1 z}+i B_{1 x} B_{0 y}^{\prime} /\left(k B_{0 z}\right),
$$

such that the residual $B_{1 z}-Q$ is the even parity extension into the boundary layer of the functional form of the outer solution, linked to $B_{1 x}$. Using the above discussed simplifications based only on the application to a short-scale boundary layer around $x=0$, introducing the equilibrium magnetic gradient scale length $L_{B} \equiv L / \epsilon_{B}$, and in terms of $Q$ as primary variable instead of $B_{1 z}$, our basic linear system Eqs.(25)-(28) becomes

$$
\begin{gathered}
\left(\beta+\epsilon_{\gamma}^{2}\right) \frac{\rho_{1}}{\rho_{0}}=-\epsilon_{\gamma}^{2} \xi^{\prime}-\frac{Q}{B_{0}}, \\
\epsilon_{\eta} \frac{B_{1 x}^{\prime \prime}}{k^{2} B_{0}}=\epsilon_{\gamma}\left(\frac{B_{1 x}(0)}{B_{0}}-\frac{x}{L_{B}} i k \xi\right)-\alpha \frac{x}{L_{B}} \frac{Q}{B_{0}}, \\
\frac{\epsilon_{\gamma}^{2} \beta}{\beta+\epsilon_{\gamma}^{2}} \xi^{\prime \prime}-i \frac{x}{L_{B}} \frac{B_{1 x}^{\prime \prime}}{k B_{0}}=\frac{\epsilon_{\gamma}^{2}}{\beta+\epsilon_{\gamma}^{2}} \frac{Q^{\prime}}{B_{0}}, \\
\epsilon_{\eta} \epsilon_{\gamma} \frac{Q^{\prime \prime}}{k^{2} B_{0}}-\left(\frac{x^{2}}{L_{B}^{2}}+\epsilon_{\gamma}^{2} \frac{1+\beta+\epsilon_{\gamma}^{2}}{\beta+\epsilon_{\gamma}^{2}}\right) \frac{Q}{B_{0}}+\epsilon_{\gamma} \alpha \frac{x}{L_{B}} \frac{B_{1 x}^{\prime \prime}}{k^{2} B_{0}}= \\
\epsilon_{\gamma} \frac{x}{k L_{B}^{2}}\left(\epsilon_{\gamma} k \xi-i \alpha \frac{Q}{B_{0}}\right)+\frac{\epsilon_{\gamma}^{4} \xi^{\prime}}{\beta+\epsilon_{\gamma}^{2}},
\end{gathered}
$$

where $B_{1 x}^{\prime \prime}$ can be eliminated algebraically by substituting Eq. (41) into Eqs. (42) and (43). Thus, 
the explicit calculations of $\rho_{1}$ and $B_{1 x}^{\prime}$ become decoupled from the pair of equations that determine $\xi$ and $Q$, and can be carried out a posteriori once the latter have been solved for.

Equations (40)-(43) represent the most general inner system that follows from the original linear set, (11)-(17), under the sole assumption of a short-scale layer around $x=0$ for $\epsilon_{\eta} \ll 1$. This system will be further reduced by implementing the following orderings that constitute our main working hypotheses:

$$
\begin{gathered}
\epsilon_{B}=O(1), \quad k L=O(1)\left[k^{-1} \Delta^{\prime}=O(1)\right] . \\
\beta \gg O\left(\epsilon_{\gamma}^{2}\right), \\
\alpha \leq O\left(\epsilon_{\eta}^{-1 / 5}\right) .
\end{gathered}
$$

As mentioned earlier, these orderings allow arbitrary magnitudes of the magnetic guide field and all practical values of the Hall parameter, and exclude only extremely low betas and very large values of $k^{-1} \Delta^{\prime}$. The condition (45) on $\beta$ limits our analysis to subsonic tearing mode growths, $\gamma \ll k c_{s}$, which is largely the sole case of practical interest. Sonic or supersonic growths would only be approached in the excluded regimes of extremely low $\beta$ or very large $k^{-1} \Delta^{\prime}$, which are not relevant to most realistic applications. The solutions to be obtained will also confirm that the condition (46) on $\alpha$ allows the consistent neglect of the right-hand sides of Eqs. (42) and (43). With these simplifications, these equations become

$$
\begin{gathered}
\frac{\epsilon_{\gamma} \epsilon_{\eta}}{k} i \xi^{\prime \prime}=\frac{x}{L_{B}}\left(-\frac{B_{1 x}(0)}{B_{0}}+\frac{x}{L_{B}} i k \xi+\frac{\alpha}{\epsilon_{\gamma}} \frac{x}{L_{B}} \frac{Q}{B_{0}}\right), \\
\epsilon_{\eta} \epsilon_{\gamma} \frac{Q^{\prime \prime}}{k^{2} B_{0}}=\left(\frac{x^{2}}{L_{B}^{2}}+\epsilon_{\gamma}^{2} \frac{1+\beta}{\beta}\right) \frac{Q}{B_{0}}+\frac{\epsilon_{\gamma}^{3} \alpha}{k} i \xi^{\prime \prime} .
\end{gathered}
$$

Equations (47) and (48) yield real solutions for $i \xi / B_{1 x}(0)$ and $Q / B_{1 x}(0)$ and, as a consequence, Eqs. (36) and (41) yield a real $\epsilon_{\gamma}$. Any of the terms on the right-hand sides of Eqs. (42) and 
(43) would make $i \xi / B_{1 x}(0)$ or $Q / B_{1 x}(0)$ complex, resulting in a complex growth rate. Thus, our condition (46) on $\alpha$ ensures that the tearing mode is purely-growing.

The last step is to write the above inner system in dimensionless form. To this effect we introduce the characteristic length $d_{0}$ defined by

$$
d_{0}=L_{B}^{1 / 2}\left(d_{\eta} d_{\gamma}\right)^{1 / 4}=\left(\epsilon_{\gamma} \epsilon_{\eta}\right)^{1 / 4}\left(L_{B} / k\right)^{1 / 2}
$$

the dimensionless variables

$$
\bar{x}=\frac{x}{d_{0}}, \quad \bar{\xi}=\frac{d_{0} B_{0}}{L_{B} B_{1 x}(0)} i k \xi, \quad \bar{Q}=\frac{d_{0} \alpha}{L_{B} \epsilon_{\gamma}} \frac{Q}{B_{1 x}(0)},
$$

and the dimensionless parameters

$$
\sigma=\alpha \epsilon_{\gamma}^{1 / 2} \epsilon_{\eta}^{-1 / 2}, \quad \tau=\left(\beta^{-1}+1\right) \epsilon_{\gamma}^{3 / 2} \epsilon_{\eta}^{-1 / 2} k L_{B}
$$

In terms of these, Eqs. (47) and (48) become

$$
\begin{gathered}
\frac{d^{2} \bar{\xi}}{d \bar{x}^{2}}=\bar{x}^{2}(\bar{\xi}+\bar{Q})-\bar{x}, \\
\frac{d^{2} \bar{Q}}{d \bar{x}^{2}}=\left(\bar{x}^{2}+\tau\right) \bar{Q}+\sigma^{2} \frac{d^{2} \bar{\xi}}{d \bar{x}^{2}},
\end{gathered}
$$

which constitute the fundamental inner tearing mode system for our present two-fluid analysis. The definitions (50) and (51) of dimensionless inner variables and parameters rely on the idea that inertial and resistive effects establish the basic natural scales for the tearing mode system, (52) and (53). There, the terms proportional to $\sigma^{2}$ and $\tau$ measure the Hall and $\beta$ (i.e compressibility) effects, respectively. The mathematical scaling parameters $\sigma^{2}$ and $\tau$ involve the yet to be determined $\epsilon_{\gamma}$, so we have to wait until the mode growth rate has been found to define the relevant two basic input parameters in terms of the primary dimensionless data of Eq. (30). The solution of equations (52) 
and (53) yields $\bar{\xi}$ and $\bar{Q}$ as real, odd functions of $\bar{x}$, depending parametrically on $\sigma$ and $\tau$. Their boundary conditions are $\bar{\xi}=\bar{Q}=0$ at $\bar{x}=0$ and

$$
\bar{\xi}=\bar{x}^{-1}+2\left(1+\sigma^{2}\right) \bar{x}^{-5}+\ldots, \quad \bar{Q}=-2 \sigma^{2} \bar{x}^{-5}+\ldots, \quad \text { for } \quad \bar{x} \gg\left\{1, \tau^{1 / 2}\right\}
$$

\section{Inner region solution in different parametric regimes}

The inner tearing mode solution depends on the two dimensionless parameters $\sigma$ and $\tau$ defined in Eq. (51). Seven parametric regions (PR0, PR1,..,PR6) can be distinguished in a $(\sigma, \tau)$ plane, as sketched in Fig. 2. There is the parametric region PR0, with $\sigma=O(1)$ and $\tau=O(1)$, where the full equations (52) and (53) have to be solved numerically. In the other six regions, these equations admit some asymptotic reduction. In some of them, the inner boundary layer splits in two asymptotic sublayers: an intermediate, non-resistive layer of characteristic width $d_{2}$ and an innermost, resistive layer of width $d_{1} \ll d_{2}$. When this is the case, the length $d_{0}$, Eq. (49), is the geometric mean of the two sublayer lengths, $d_{0}=\sqrt{d_{1} d_{2}}$. Otherwise, these two layers merge into a single one of width $d_{0}$. The characteristics of the tearing mode solutions in each of these parametric regions are summarized in table 2.

Before proceeding with the specific asymptotic solutions for the different parametric regimes (PR1,..,PR6), some general features of our tearing mode system, Eqs. (52) and (53), are worth commenting on. First, if we drop all second-order derivatives in Eqs. (52) and (53), we are neglecting all resistive and dynamic effects and recover the asymptotic form of the outer solution, $\bar{Q}=0$ and $\bar{\xi}=1 / \bar{x}$. Second, dropping the LHS of Eqs. (52) and (53) corresponds to neglecting just the resistivity effects and leads to

$$
\frac{d^{2} \bar{\xi}}{d \bar{x}^{2}}=\frac{\left(\bar{x}^{2}+\tau\right)\left(\bar{x}^{2} \bar{\xi}-\bar{x}\right)}{\sigma^{2} \bar{x}^{2}}=-\bar{Q} \frac{\bar{x}^{2}+\tau}{\sigma^{2}}
$$

which are the equations for the intermediate layer whenever it exists independently of the innermost 
resistive layer. Third, dropping only the LHS of Eq. (53) leads to

$$
\frac{d^{2} \bar{\xi}}{d \bar{x}^{2}}=\frac{\left(\bar{x}^{2}+\tau\right)\left(\bar{x}^{2} \bar{\xi}-\bar{x}\right)}{\left(1+\sigma^{2}\right) \bar{x}^{2}+\tau}=-\bar{Q} \frac{\bar{x}^{2}+\tau}{\sigma^{2}}
$$

which is the model of the resistive layer when the diffusion of $B_{1 z}$ is negligible. Notice that $d^{2} \bar{\xi} / d \bar{x}^{2}$ is involved both in the resistive diffusion of $B_{1 x}$ and the Hall effects; the differences between Eqs.(55) and (56) identify the terms related to the diffusion of $B_{1 x}$. The detailed analysis of each of the six parametric regions $(\mathrm{PR} 1, \ldots, \mathrm{PR} 6)$ amenable to asymptotic reduction follows next.

\subsection{Weak-Hall regime (PR1)}

The regime to be labeled PR1 spans the asymptotic region where Hall effects are negligible, which corresponds to $\sigma^{2} \ll 1$ or $\sigma^{2} \ll \tau$. There, a solution with $\bar{x} \sim 1, \bar{\xi} \sim 1$ and $\bar{Q} \sim \sigma^{2} /(1+\tau) \ll 1$ is obtained. Hence, the contribution of $B_{1 z}$ is negligible and the inner tearing mode system reduces to the classic single-fluid equation [1], independent of $\sigma$ and $\tau$ :

$$
d^{2} \bar{\xi} / d \bar{x}^{2}-\bar{x}^{2} \bar{\xi}=-\bar{x}
$$

whose well known solution is the parabolic cylinder function $[1,8] \xi(\bar{x})=U(0, \bar{x}) \equiv F(\bar{x})$ :

$$
F(\bar{x})=\frac{\bar{x}}{2} \int_{0}^{1} d \mu\left(1-\mu^{2}\right)^{-1 / 4} \mathrm{e}^{-\mu \bar{x}^{2} / 2}
$$

The corresponding dispersion relation, to be discussed in Section 5, is the same as in the single-fluid theory $[1]$.

\subsection{General high-beta regime (PR2)}

This region corresponds to sufficiently high values of $\beta$ to make $\tau \ll 1$, so that with a negligible $\tau$ the results become independent of $\beta$, compressible effects remain negligible and the length scale associated with two-fluid effects is the ion skin depth $d_{i}$. The two-fluid tearing system depends only on the parameter $\sigma$, which is here assumed to be arbitrary and formally ordered as $\sigma=O(1)$. 
The inner tearing mode system for this regime is the $\tau \rightarrow 0$ limit of our Eqs. (52) and (53):

$$
\begin{aligned}
& \frac{d^{2} \bar{\xi}}{d \bar{x}^{2}}=\bar{x}^{2}(\bar{\xi}+\bar{Q})-\bar{x} \\
& \frac{d^{2} \bar{Q}}{d \bar{x}^{2}}-\sigma^{2} \frac{d^{2} \bar{\xi}}{d \bar{x}^{2}}=\bar{x}^{2} \bar{Q}
\end{aligned}
$$

which indicates that the resistive diffusions of both $B_{1 x}$ and $B_{1 z}$ are relevant. This system is diagonalized by the eigenfunction linear combinations

$$
V_{n}=\bar{\xi}+a_{n} \bar{Q} \quad n=1,2,
$$

with

$$
a_{n}(\sigma)=\frac{1}{2}+\frac{(-1)^{n}}{2}\left(1+\frac{4}{\sigma^{2}}\right)^{1 / 2}
$$

in terms of which it becomes

$$
\frac{1}{\lambda_{n}} \frac{d^{2} V_{n}}{d \bar{x}^{2}}=\bar{x}^{2} V_{n}-\bar{x}
$$

where the eigenvalues $\lambda_{n}$ are

$$
\lambda_{n}(\sigma)=1+\sigma^{2} a_{n}(\sigma)
$$

and satisfy $\lambda_{1} \lambda_{2}=1$. From Eq. (54), the boundary conditions for $V_{n}$ are $V_{n}(0)=0$ and $V_{n}(|\bar{x}| \gg$ 1) $=\bar{x}^{-1}+O\left(\bar{x}^{-5}\right)$. Upon rescaling of the variables with appropriate powers of $\lambda_{n}$, this problem becomes mathematically identical to the canonical single-fluid one, Eq. (57), and has therefore the solution

$$
V_{n}(\bar{x})=\lambda_{n}^{1 / 4} F\left(\lambda_{n}^{1 / 4} \bar{x}\right)
$$

with $F$ defined in Eq. (58). Then, the solutions for $\bar{\xi}$ and $\bar{Q}$ are

$$
\bar{\xi}(\bar{x})=\frac{a_{1} \lambda_{2}^{1 / 4} F\left(\lambda_{2}^{1 / 4} \bar{x}\right)-a_{2} \lambda_{1}^{1 / 4} F\left(\lambda_{1}^{1 / 4} \bar{x}\right)}{a_{1}-a_{2}},
$$




$$
\bar{Q}(\bar{x})=\frac{\lambda_{1}^{1 / 4} F\left(\lambda_{1}^{1 / 4} \bar{x}\right)-\lambda_{2}^{1 / 4} F\left(\lambda_{2}^{1 / 4} \bar{x}\right)}{a_{1}-a_{2}}
$$

Figure 3 shows these inner solutions for $\bar{\xi}, \bar{Q}$ and the resulting $\bar{B}_{1 y}$, for three different values of $\sigma$. For $\sigma \ll 1$ we recover the result of the weak-Hall regime and for $\sigma \gg 1$ we enter the strong-Hall, high-beta regime PR3 to be discussed next. Based on this solution, we will obtain in Section 5 a novel high-beta dispersion relation valid for arbitrary values of the Hall parameter, whose asymptots are the single-fluid dispersion relation [1] in the weak-Hall limit and a dispersion relation identical to the one obtained, under different assumptions, with the so-called electron-MHD model $[3,4]$ in the strong-Hall limit.

\subsection{Strong-Hall, high-beta regime (PR3)}

This parametric region is defined by the conditions $\sigma \gg 1$ and $\tau \ll \sigma$, corresponding to the large- $\sigma$ or strong-Hall limit of the previous high-beta regime PR2. For $\sigma \gg 1$, Eqs. (62), (64), (66), and (67) show that the non-ideal boundary layer splits into two distinct asymptotic sublayers: an innermost sublayer of width $d_{1}=d_{0} \lambda_{1}^{-1 / 4}=d_{0} \sigma^{-1 / 2}$ and an intermediate sublayer of width $d_{2}=d_{0} \lambda_{2}^{-1 / 4}=d_{0} \sigma^{1 / 2}$, that is

$$
d_{1}=\sqrt{\frac{L_{B} d_{\eta}}{k d_{i}}}, \quad d_{2}=\sqrt{k d_{i} L_{B} d_{\gamma}}
$$

These expressions make clear the role of the ion-skin-depth, $d_{i}$, in separating these two scales. The absence of $d_{\eta}$ in $d_{2}$ is a hint that the intermediate layer is non-resistive.

Although the general solution given by Eqs.(66)-(67) contains this strong-Hall limit where the two sublayers separate, it is useful to show explicitly the asymptotic models for each of these sublayers. In the intermediate sublayer where the resistive diffusion of both $B_{1 z}$ and $B_{1 x}$ is negligible, the scaling of variables is

$$
\bar{x} \sim \sigma^{1 / 2}, \quad \bar{Q} \sim \bar{\xi} \sim \sigma^{-1 / 2}
$$


and the reduced system is the $\tau \rightarrow 0$ limit of Eq. (55):

$$
\sigma^{2} \frac{d^{2} \bar{\xi}}{d \bar{x}^{2}}=\bar{x}^{2} \bar{\xi}-\bar{x}=-\bar{x}^{2} \bar{Q}
$$

In the innermost sublayer, the scaling of the variables is

$$
\bar{x} \sim \sigma^{-1 / 2}, \quad \bar{Q} \sim \sigma^{1 / 2}, \quad \bar{\xi} \sim \sigma^{-3 / 2}
$$

and our tearing system, Eqs.(52)-(53), reduces to

$$
d^{2} \bar{Q} / d \bar{x}^{2}=\sigma^{2}\left(\bar{x}^{2} \bar{Q}-\bar{x}\right)=\sigma^{2} d^{2} \bar{\xi} / d \bar{x}^{2}
$$

Thus, in contrast to the weak-Hall regime where $\bar{Q}$ is negligible and $\bar{\xi}$ is bounded by the diffusion of $B_{1 x}$, in this PR3 regime the $B_{1 x}$ diffusion is negligible in the intermediate sublayer where the Hall effects give rise to $\bar{Q}$ and bound $\bar{\xi}$. However, the intermediate sublayer solution for $\bar{Q}$, Eq. (70), is unbounded as $\bar{x} \rightarrow 0$. This is regularized by the diffusion of $B_{1 x}$ and $B_{1 z}$ in the innermost, resistive sublayer governed by Eq. (72). As mentioned earlier, the dispersion relation to be found for this high-beta, strong-Hall regime will turn out to be same as the one obtained in a different context with the so-called electron-MHD model [3, 4].

\subsection{General strong-Hall regime (PR4)}

The parametric region labeled (PR4) corresponds to $\tau \sim \sigma \gg 1$ and has been studied by Mirnov et al.[2] for $\epsilon_{B} \ll 1$. This regime preserves the same scaling of variables as in PR3, with two sublayers having the characteristic widths defined in Eq. (68). The intermediate layer has the scalings of Eq. (69) and is governed by Eq. (55). Following the Fourier transform method of [9], this system admits a solution in terms of parabolic cylinder functions[8]. Thus we obtain the intermediate layer solution:

$$
\bar{\xi}(\bar{x})=\frac{1}{\bar{x}}-\bar{Q}(\bar{x})=\frac{1-\sigma}{2 \sigma} \int_{0}^{+\infty} d k \sin k \bar{x} \frac{U(\tau / 2 \sigma, \sqrt{2 \sigma} k)}{U(\tau / 2 \sigma, 0)}+\tau \int_{0}^{+\infty} d k \frac{\sin k \bar{x}}{\tau+k^{2} \sigma^{2}} .
$$


The scalings of Eq. (71) hold in the innermost resistive layer, where the governing system becomes

$$
\begin{gathered}
d^{2} \bar{Q} / d \bar{x}^{2}=\left(\sigma^{2} \bar{x}^{2}+\tau\right) \bar{Q}-\sigma^{2} \bar{x}, \\
d^{2} \bar{\xi} / d \bar{x}^{2}=\left(\bar{x}^{2} \bar{Q}-\bar{x}\right)
\end{gathered}
$$

and we obtain the resistive layer solution [2]

$$
\bar{Q}(\bar{x})=\sigma \int_{0}^{+\infty} d k \sin k \bar{x} \frac{U(\tau / 2 \sigma, \sqrt{2 \sigma} k)}{U(\tau / 2 \sigma, 0)}
$$

with $\bar{\xi} \sim \bar{Q}^{-3} \ll \bar{Q}$ which can be integrated after Eqs. (75) and (76) but will not contribute to the leading order dispersion relation. The $\tau \ll \sigma$ and $\tau \gg \sigma$ limits of these solutions correspond, respectively, to the previous PR3 and to the strong-Hall, low-beta parametric regime PR5 to be considered next.

\subsection{Strong-Hall, low-beta regime (PR5)}

This regime is defined by the conditions $1 \ll \sigma \ll \tau \ll \sigma^{2}$. Here, the $\tau \gg \sigma$ limit produces qualitative changes in the PR4 solution and the lengths of the intermediate and resistive sublayers become respectively

$$
d_{2}=d_{s}, \quad d_{1}=\frac{L_{B} \sqrt{d_{\gamma} d_{\eta}}}{d_{s}}
$$

where $d_{s}=d_{i} \sqrt{\beta}$ is the ion sound gyroradius. The scaling of variables in the intermediate, nondiffusive layer of PR5 is

$$
\bar{x} \sim \tau^{-1 / 2} \sigma, \quad \bar{Q} \sim \bar{\xi} \sim \tau^{1 / 2} \sigma^{-1},
$$

and the system (55) reduces to

$$
\frac{d^{2} \bar{\xi}}{d \bar{x}^{2}}=\frac{\tau}{\sigma^{2}}\left(\bar{\xi}-\frac{1}{\bar{x}}\right)=-\frac{\tau}{\sigma^{2}} \bar{Q}
$$


which has the solution

$$
\bar{\xi}(\bar{x})=\frac{1}{\bar{x}}-\bar{Q}(\bar{x})=\tau \int_{0}^{+\infty} d k \frac{\sin k \bar{x}}{\tau+k^{2} \sigma^{2}} .
$$

The innermost, resistive layer has the scalings

$$
\bar{x} \sim \tau^{1 / 2} \sigma^{-1}, \quad \bar{Q} \sim \tau^{-1 / 2} \sigma, \quad \bar{\xi} \sim \tau^{3 / 2} \sigma^{-3},
$$

which make the $d^{2} \bar{Q} / d \bar{x}^{2}$ term of Eq. (74) negligible, leading to the simple solution

$$
\bar{Q}=\frac{\sigma^{2} \bar{x}}{\sigma^{2} \bar{x}^{2}+\tau}
$$

with $\bar{\xi} \sim \bar{Q}^{-3} \ll \bar{Q}$ determined by

$$
\frac{d^{2} \bar{\xi}}{d \bar{x}^{2}}=\left(\bar{x}^{2} \bar{Q}-\bar{x}\right)=-\frac{\tau \bar{x}}{\sigma^{2} \bar{x}^{2}+\tau} .
$$

Thus, the intermediate layer solution here has the same features as the one found in PR3 and PR4: $\bar{\xi}$ remains bounded by $\bar{Q}$, which is now triggered by Hall and compressibility effects and is unbounded in its small $\bar{x}$ limit. On the other hand, the behavior of the resistive layer of PR5 is peculiar. The $d^{2} \bar{Q} / d \bar{x}^{2}$ term in Eq. (74), that represents the resistive diffusion of $B_{1 z}$, is negligible and $\bar{Q}$ is regularized by a combination of compressibility and $B_{1 x}$-diffusion. The latter manifests itself through the $d^{2} \bar{\xi} / d \bar{x}^{2}$ term of Eq. (75), which is not negligible even though the magnitude of $\bar{\xi}$ is negligible within this resistive layer. This strong-Hall, low-beta regime is the only case with two distinct sublayers but negligible $B_{1 z}$-diffusion.

A unified treatment of the two sublayers relevant to this low-beta, strong-Hall regime is possible once we know that the $B_{1 z}$-diffusion is negligible and that the defining conditions and $\bar{x}$ scalings [Eqs. (78),(81)] of PR5 guarantee that $\sigma^{2} \gg 1$ and $\tau \gg \bar{x}^{2}$ through the two sublayers. Then, the $\sigma^{2} \gg 1$ and $\tau \gg \bar{x}^{2}$ limit of Eq. (56),

$$
\frac{d^{2} \bar{\xi}}{d \bar{x}^{2}}=\frac{\bar{x}^{2} \bar{\xi}-\bar{x}}{\left(\sigma^{2} / \tau\right) \bar{x}^{2}+1}=-\frac{\tau}{\sigma^{2}} \bar{Q},
$$


provides the unified system that covers the two previously given sublayer systems of Eqs.(79) and (83). Equation (84) is the "constant- $B_{1 x}($ constant- $\psi)$ approximation" version of the system considered by Kuvshinov[7] in his study of so called semi-collisional tearing modes. In our case, the separation into two distinct asymptotic sublayers is a consequence of the smallness of $\tau / \sigma^{2}$ and we obtain a simpler solution, i.e. Eq. (82), that will still yield the same semi-collisional tearing mode dispersion relation.

\subsection{General low-beta regime (PR6)}

This regime corresponds to $\tau \sim \sigma^{2} \gg 1$, with the scalings $\bar{x} \sim \bar{\xi} \sim \bar{Q} \sim 1$. Accordingly, the inner region consists of a single layer governed by the system given in Eq. (84), with the parameter $\tau / \sigma^{2}$ as well as all the variables ordered as comparable to unity. At the asymptotic ends of this regime, the $\tau / \sigma^{2} \ll 1$ limit corresponds to the previously discussed PR5 where the inner region separates into two distinct sublayers, and the $\tau / \sigma^{2} \rightarrow \infty$ (hence $\bar{Q} \rightarrow 0$ ) limit corresponds to the weak-Hall regime governed by Eq. (57). Therefore, the PR6 regime provides the continuous transition between PR5 and PR1, which completes our covering of the parameter space.

An analytic solution of Eq. (84) in terms of standard mathematical functions appears to be unavailable when $\tau / \sigma^{2} \sim 1$. Instead, we have carried out a direct numerical integration (matching the simple asymptotic behavior at $\bar{x} \gg 1$ ) and Figure 4 shows the solutions for three different values of $\tau / \sigma^{2}$.

\section{The dispersion relation}

Once $\bar{\xi}$ and $\bar{Q}$ are known, the integration of Eq. (41) yields

$$
\frac{d B_{1 x}}{d x}=B_{1 x}(0) k^{2} d_{0} \frac{\epsilon_{\gamma}}{\epsilon_{\eta}} \int_{0}^{x / d_{0}}(1-\bar{x} \bar{\xi}-\bar{x} \bar{Q}) d \bar{x}
$$

and the asymptotic matching of this result with Eq. (36) yields the dispersion relation

$$
k^{-1} \Delta^{\prime}=\epsilon_{\gamma}^{5 / 4} \epsilon_{\eta}^{-3 / 4}\left(k L_{B}\right)^{1 / 2} D(\sigma, \tau),
$$


where

$$
D(\sigma, \tau)=\int_{-\infty}^{+\infty}(1-\bar{x} \bar{\xi}-\bar{x} \bar{Q}) d \bar{x}
$$

Equation (86) specifies the dimensionless normalized growth rate $\epsilon_{\gamma}$ as an implicit function of the five dimensionless input parameters $\epsilon_{\eta}, \alpha, \beta, k L_{B}=\epsilon_{B}^{-1} k L$ and $k^{-1} \Delta^{\prime}(k L)$ :

$$
\frac{\epsilon_{\gamma}^{5 / 4}\left(k L_{B}\right)^{1 / 2}}{\epsilon_{\eta}^{3 / 4} k^{-1} \Delta^{\prime}} D\left(\frac{\epsilon_{\gamma}^{1 / 2} \alpha}{\epsilon_{\eta}^{1 / 2}}, \frac{\epsilon_{\gamma}^{3 / 2} k L_{B}(1+\beta)}{\epsilon_{\eta}^{1 / 2} \beta}\right)=1
$$

The inner layer solutions for $\bar{\xi}(\bar{x})$ and $\bar{Q}(\bar{x})$ obtained in the previous section allow us to derive the different expressions of the dispersion function $D(\sigma, \tau)$, Eq. (87), that apply in the different parametric regions. In the weak-Hall regime PR1, $D(\sigma, \tau)$ is constant:

$$
D(\sigma, \tau)=\int_{-\infty}^{+\infty}[1-y F(y)] d y=\frac{2 \pi \Gamma(3 / 4)}{\Gamma(1 / 4)} \equiv C \simeq 2.12
$$

In the general high-beta regime PR2, the dispersion function takes the form $D(\sigma, \tau)=C f_{2}(\sigma)$, where

$$
f_{2}(\sigma)=\frac{a_{1} \lambda_{1}^{-1 / 4}-a_{2} \lambda_{2}^{-1 / 4}}{a_{1}-a_{2}}
$$

and $a_{n}(\sigma)$ and $\lambda_{n}(\sigma)$ are specified in Eqs. (62) and (64). Substituting these, we get the explicit form

$$
f_{2}(\sigma)=\frac{1}{2} \sum_{n=1}^{2}\left[1+(-1)^{n}\left(1+4 / \sigma^{2}\right)^{-1 / 2}\right]\left[1+\sigma^{2} / 2+(-1)^{n} \sigma\left(1+\sigma^{2} / 4\right)^{1 / 2}\right]^{-1 / 4}
$$

The function $f_{2}(\sigma)$ is plotted in Figure 5 . We have $f_{2}(0)=1$ which corresponds to the overlapping with PR1, $f_{2}(1)=0.92$, and $f_{2}(\sigma \gg 1) \simeq \sigma^{-1 / 2}$ which corresponds to the overlapping with PR3. In PR3 where $\sigma \gg 1$, the contribution to the dispersion function of the innermost resistive layer is of order $\sigma^{-1 / 2}$ and dominates over the contribution of the intermediate layer of order $\sigma^{-3 / 2}$. In this sense, it can be said that the tearing-mode growth in PR3 is dominated by the $B_{1 z}$-diffusion.

In order to determine the dispersion function in PR4 (and PR5) we take into account the fact 
that the dominant contribution comes from the innermost resistive layer. Then, using Eq. (76),

$$
1-\bar{x} \bar{\xi}-\bar{x} \bar{Q} \simeq 1-\bar{x} \bar{Q}=\sqrt{2 \sigma} \int_{0}^{\infty} \cos k \bar{x} \frac{U^{\prime}(\tau / 2 \sigma, \sqrt{2 \sigma} k)}{U(\tau / 2 \sigma, 0)} d k
$$

which yields $D(\sigma, \tau)=C \sigma^{-1 / 2} f_{4}(\tau / \sigma)$ with

$$
f_{4}(u)=-\frac{\sqrt{2} \pi}{C} \frac{U^{\prime}(u / 2,0)}{U(u / 2,0)}=\frac{2 \pi}{C} \frac{\Gamma[(3+u) / 4]}{\Gamma[(1+u) / 4]}
$$

Figure 5 depicts the function $f_{4}(u)$. We have $f_{4}(0)=1$ which corresponds to the overlapping with PR3 and $f_{4}(u \gg 1) \simeq \pi \sqrt{u} / C$ which corresponds to the overlapping with PR5.

In PR6, the dispersion function takes the form $D(\sigma, \tau)=C f_{6}\left(\sigma^{2} / \tau\right)$, where the function $f_{6}(u)$ is obtained numerically and is shown in Fig. 5, together with the approximate, simple fit

$$
f_{6}(u) \simeq \frac{1-u / 4+\pi u^{2} / 20}{1+C u^{5 / 2} / 20}
$$

We have $f_{6}(0)=1$ which corresponds to the overlapping with PR1 and $f_{6}(u \gg 1)=\pi /(C \sqrt{u})$ which corresponds to the overlapping with PR5.

The simple asymptotic expressions of $f_{2}, f_{4}$, and $f_{6}$ for small and large arguments, allow us to recover explicit forms of the dispersion relation in PR1 PR3 and PR5. Thus, in the weak-Hall regime PR1 we have

$$
\epsilon_{\gamma}=\epsilon_{\eta}^{3 / 5}\left(\frac{\epsilon_{B} \Delta^{\prime 2}}{C^{2} k^{3} L}\right)^{2 / 5}
$$

which coincides with the classic single-fluid dispersion relation [1]. In the strong-Hall, high-beta regime PR3 we have

$$
\epsilon_{\gamma}=\epsilon_{\eta}^{1 / 2}\left(\alpha \frac{\epsilon_{B} \Delta^{\prime 2}}{C^{2} k^{3} L}\right)^{1 / 2}
$$

which coincides with the dispersion relation obtained with the so-called electron MHD model [3, 4]. 
Finally, in the strong-Hall, low-beta regime PR5 (where $\beta \ll 1$ ) we have

$$
\epsilon_{\gamma}=\epsilon_{\eta}^{1 / 3}\left(\alpha \beta^{1 / 2} \frac{\epsilon_{B} \Delta^{\prime}}{\pi k^{2} L}\right)^{2 / 3}
$$

which coincides with the so-called semicollisional tearing mode dispersion relation [7]. We recall that $\alpha=k d_{i}$ and $\alpha \beta^{1 / 2}=k d_{s}$, so the characteristic lengths associated with two-fluid effects in PR3 and PR5 are respectively the ion skin depth and the ion sound gyroradius. The complete functions $f_{2}, f_{4}$ and $f_{6}$ provide the smooth connections between these three classic dispersion relations in the transitional regimes PR2, PR4 and PR6. The exact analytic result for PR2 is a novel contribution of the present work. The result for PR4 coincides with the corresponding analytic result derived in Ref.[2] assuming a strong magnetic guide field $\left(\epsilon_{B} \ll 1\right)$, but is shown here to apply also for $\epsilon_{B} \sim 1$. The transition between the semicollisional and single-fluid dispersion relations in PR6 is not covered by the analyses of either Ref.[2] or Ref.[7] and our semi-analytic treatment of this regime is also new.

\section{Concluding discussion}

The complete form of our general dispersion relation Eq. (88) involves only three independent combinations of the eigenvalue $\epsilon_{\gamma}$ and the five dimensionless input parameters of the problem. It is therefore appropriate to introduce

$$
\hat{\gamma}=\frac{\epsilon_{\gamma}}{\epsilon_{\eta}^{3 / 5}}\left(\frac{C^{2} k^{3} L}{\epsilon_{B} \Delta^{\prime 2}}\right)^{2 / 5}, \quad \hat{\alpha}=\frac{\alpha}{\epsilon_{\eta}^{1 / 5}}\left(\frac{\epsilon_{B} \Delta^{\prime 2}}{C^{2} k^{3} L}\right)^{1 / 5}, \quad \hat{\beta}=\frac{\beta}{\epsilon_{\eta}^{2 / 5}(1+\beta)}\left(\frac{C^{8} \epsilon_{B} k^{2}}{\pi^{5} L \Delta^{\prime 3}}\right)^{2 / 5}
$$

as the naturally scaled growth rate output and the two naturally scaled input parameters, respectively. In terms of these, the general dispersion relation assumes the simple reduced form

$$
\hat{\gamma}^{5 / 4} D\left(\hat{\gamma}^{1 / 2} \hat{\alpha}, \frac{C^{2} \hat{\gamma}^{3 / 2}}{\pi^{2} \hat{\beta}}\right)=C
$$


and its explicit asymptotic expressions for the regimes PR1, PR3 and PR5 become simply

$$
\hat{\gamma}(\hat{\alpha}, \hat{\beta})= \begin{cases}1 & \text { in PR1 } \\ \hat{\alpha}^{1 / 2} & \text { in PR3 } \\ \left(\hat{\alpha} \hat{\beta}^{1 / 2}\right)^{2 / 3} & \text { in PR5 }\end{cases}
$$

Again, the connections between these three asymptotic expressions through the transitional parametric regions PR2, PR4 and PR6 are provided by our corresponding forms of the dispersion function $\mathrm{D}$ determined with the functions $f_{2}, f_{4}$, and $f_{6}$.

Once the growth rate has been solved for, the parametric region boundaries (that were initially established in terms of the mathematical scaling parameters $\sigma$ and $\tau$ ) can now be defined in terms of the primary input parameters $\hat{\alpha}$ and $\hat{\beta}$ alone. The result is given in Table 2 and shown graphically in Fig.6. The surface $\hat{\gamma}(\hat{\alpha}, \hat{\beta})$ representing our reduced form of the dispersion relation Eq. (97) is shown in Fig.7 where, for the sake of simplicity, simple patchings at lines representing the transition regions PR2, PR4 and PR6 have been used when generating the plot. The largest normalized growth rates are found in the strong-Hall, high-beta region PR3.

Our working hypotheses (45) and (46) limit the validity of the present analysis of subsonic, purely-growing tearing modes to a subdomain of the $(\alpha, \beta)$ plane. The corresponding restrictions can be determined in each parametric region once the growth rate solution is known. Focusing on the intermediate regions PR2, PR4 and PR6 and keeping $\epsilon_{B}$ and $k^{-1} \Delta^{\prime}$ of order unity [Eq. (44)], it turns out that a subsonic, purely-growing tearing mode exists: without any additional restriction in PR2; for $\beta \gg \epsilon_{\eta}^{2 / 3}$ (or equivalently $\alpha \ll \epsilon_{\eta}^{-1 / 3}$ ) in PR4; and for $\beta \gg \epsilon_{\eta}^{6 / 5}$ (or equivalently $\left.\alpha \ll \epsilon_{\eta}^{-1 / 5}\right)$ in PR6. Furthermore, the transition to a transonic tearing mode in both PR4 and PR6 takes place simultaneously to the transition to an oscillatory (unstable) tearing mode. This suggests that supersonic (and cold plasma) tearing modes would not be purely growing.

Except for $k L$ close to one, the tearing instability index, Eq. (37), can be approximated by $k^{-1} \Delta^{\prime} \simeq 2(k L)^{-2}$. Using this approximation and also $\beta /(1+\beta) \simeq \beta$, we obtain scaling laws for the tearing mode growth rate in the regions PR1, PR3, and PR5 as given in Table 3. The extension to the intermediate regions, by patching where these laws intersect, is straightforward. Considering 
the dependence on $k^{-1} \Delta^{\prime}, \hat{\beta}$ and $\hat{\alpha}^{-2}$ are both proportional to $k \Delta^{\prime-1}$ and $\epsilon_{\gamma}$ is proportional to $k^{-1} \Delta^{\prime} \hat{\gamma}(\hat{\alpha}, \hat{\beta})$. Therefore, as $k^{-1} \Delta^{\prime}$ increases, $\hat{\beta} \hat{\alpha}^{2}$ remains constant and the parametric space point either remains always in PR1, moves from PR1 to PR6 through PR0, or moves from PR1 to PR5 through PR3. This last case is favored except at very low $\beta$ as illustrated in Fig. 8 for the two tokamak plasmas of Table 1. Notice that our novel solution from PR1 to PR3 through PR2 [Eqs. (66), (67), and (90)] covers well the $k^{-1} \Delta^{\prime}=O(1)$ range for these tokamaks. Considering the dependence on $\epsilon_{B}, \hat{\beta}$ and $\hat{\alpha}^{2}$ are proportional to $\epsilon_{B}^{2 / 5}$ and $\epsilon_{\gamma}$ is proportional to $\epsilon_{B}^{2 / 5} \hat{\gamma}(\hat{\alpha}, \hat{\beta})$ so that the parametric space point tends to move towards PR1 as $\epsilon_{B}$ decreases. Therefore, the two-fluid theory is more appropriate for $\epsilon_{B}=O(1)$.

The scaling laws for the widths of the whole non-ideal region and the resistive sublayer are also given in Table 3. There, taking into consideration the boundaries of the region PR5 we get $\left.\epsilon_{\eta}^{2 / 5} \epsilon_{B}^{-2 / 5}(k L)^{-1} \ll\left(d_{2} / L\right)\right|_{P R 5} \ll \epsilon_{\eta}^{1 / 4} \alpha^{3 / 4} \epsilon_{B}^{-1 / 4}(k L)^{-7 / 4}$, so that the width of whole non-ideal region does tend to zero when $\epsilon_{\eta} \rightarrow 0$ in all cases.

The details of the extension of the tearing mode analysis to include the finite electron inertia effect, up to the collisionless limit, are given in the Appendix. The finite electron mass increases the tearing mode growth rate relative to its massless limit, this effect becoming significant when $\hat{\alpha}^{2} \hat{\gamma}(\hat{\alpha}, \hat{\beta}) \geq O\left(m_{i} / m_{e}\right)$ which points mainly to the region PR3. Figure 8 shows the parametric point locations in the $(\hat{\alpha}, \hat{\beta})$ plane based on the physical electron to deuterium mass ratio along with those based on zero electron mass, for the Alcator C-MOD and ITER parameters of Table 1 for the range $0.1 \leq k^{-1} \Delta^{\prime} \leq 10$. Electron inertia corrections increase with $k^{-1} \Delta^{\prime}$ increasing. For the same $k^{-1} \Delta^{\prime}$, the electron inertia effect is more pronounced in Alcator C-MOD in spite of its larger resistivity because of its smaller size. At $k^{-1} \Delta^{\prime}=10$, the electron inertia figure of merit $m_{e} \gamma /\left(e^{2} n_{0} \eta\right)$ is 0.21 for Alcator C-MOD and 0.035 for ITER, resulting in growth rate increases relative to the massless limit of $6.5 \%$ and $2 \%$ respectively. For the considered Alcator C-MOD and ITER parameters, our dispersion relation would transition to an inertia-dominated regime [i.e. $\left.m_{e} \gamma /\left(e^{2} n_{0} \eta\right) \geq 1\right]$ at $k^{-1} \Delta^{\prime}$ respectively equal to 72 and 195 (i.e. $k L \simeq 1 / 6$ and $1 / 10$ ). At these points the growth rate increases relative to the massless limit would be $27 \%$ for Alcator C-MOD and $41 \%$ for ITER. 


\section{Acknowledgments}

The stay of E. Ahedo at the Massachusetts Institute of Technology was sponsored by the Ministerio de Ciencia e Innovación of Spain under Project ESP2007-62694. The work of J.J. Ramos was sponsored by the U.S. Department of Energy under Grants Nos. DEFG02-91ER54109 and DEFC02-08ER54969 at the Massachusetts Institute of Technology and as part of the author's participation in the Center for Extended MHD Modeling (CEMM).

\section{A Appendix: Generalization for finite electron inertia}

The effect of a finite but realistically small electron mass on the linear theory of the tearing mode amounts to just a slight modification of the massless electron theory presented in the main body of this paper. Due to the small electron to ion mass ratio, $m_{e} / m_{i} \ll 1$, only the inertial contribution to the electron momentum conservation equation in the non-ideal boundary layer, which like the resistive diffusivity has the character of a singular perturbation capable of breaking the magnetic frozen-in law, is of significance. So, the only modification needed in the linearized system (11)-(17) is to use, instead of Eq. (14),

$$
\boldsymbol{E}_{1}=-\boldsymbol{v}_{1} \times \boldsymbol{B}_{0}+\eta \boldsymbol{j}_{1}+\frac{m_{i}}{e}\left(\frac{\partial \boldsymbol{v}_{1}}{\partial t}+\frac{\nabla p_{i 1}}{\rho_{0}}\right)+\frac{m_{e}}{e^{2} n_{0}} \frac{\partial \boldsymbol{j}_{1}}{\partial t}
$$

Therefore, the linear tearing mode results derived for zero electron mass can be generalized to finite electron mass simply by substituting $\eta+\gamma m_{e} /\left(e^{2} n_{0}\right)$ for $\eta$. Accordingly, the general two-fluid tearing dispersion relation becomes

$$
\frac{\epsilon_{\gamma}^{5 / 4}\left(k L_{B}\right)^{1 / 2}}{\left(\epsilon_{\eta}+\epsilon_{\gamma} k^{2} d_{e}^{2}\right)^{3 / 4} k^{-1} \Delta^{\prime}} D\left(\frac{\epsilon_{\gamma}^{1 / 2} \alpha}{\left(\epsilon_{\eta}+\epsilon_{\gamma} k^{2} d_{e}^{2}\right)^{1 / 2}}, \frac{\epsilon_{\gamma}^{3 / 2} k L_{B}(1+\beta)}{\left(\epsilon_{\eta}+\epsilon_{\gamma} k^{2} d_{e}^{2}\right)^{1 / 2} \beta}\right)=1
$$

where $d_{e}^{2}=m_{e} /\left(\mu_{0} e^{2} n_{0}\right)$ is the square of the electron skin depth and the dispersion function $D(\sigma, \tau)$ is as previously derived [Eq. (87)]. 
It is worthwhile to consider the collisionless limit, where the electron inertia dominates over the resistive diffusivity as the leading effect to break the magnetic frozen-in law, since this can be the applicable regime in some situations of interest. For $\epsilon_{\eta} \ll \epsilon_{\gamma} k^{2} d_{e}^{2}$ and recalling $\alpha=k d_{i}$, Eq. (A.2) becomes

$$
\frac{\epsilon_{\gamma}^{1 / 2} L_{B}^{1 / 2}}{d_{e}^{3 / 2} \Delta^{\prime}} D\left(\frac{d_{i}}{d_{e}}, \frac{\epsilon_{\gamma} L_{B}(1+\beta)}{d_{e} \beta}\right)=1 .
$$

So, the first argument of the dispersion function is now $\sigma=d_{i} / d_{e}=\left(m_{i} / m_{e}\right)^{1 / 2} \gg 1$ and the most relevant parametric regions are likely to be the strong-Hall regimes. In fact, except for rather low values of $\beta$, the only parametric region of practical interest is PR3. Here, using the asymptotic form $D(\sigma, \tau)=C \sigma^{-1 / 2}=C\left(d_{e} / d_{i}\right)^{1 / 2}$, the dispersion relation reduces to the collisionless electron MHD form derived in Refs. [3, 4]

$$
\epsilon_{\gamma}=\frac{d_{i} d_{e}^{2} \Delta^{\prime 2}}{C^{2} L_{B}}
$$

and its validity condition is $\tau \ll \sigma$ or

$$
\frac{\beta}{1+\beta} \gg \frac{d_{e}^{2} \Delta^{\prime 2}}{C^{2}}
$$

besides the validity condition for the collisionless limit

$$
\epsilon_{\eta} \ll \frac{d_{i} d_{e}^{4} k^{2} \Delta^{\prime 2}}{C^{2} L_{B}}
$$

For sufficiently low values of beta to reach the parametric region PR5, using $\beta \ll 1$ and the asymptotic form $D(\sigma, \tau)=\pi \tau^{1 / 2} \sigma^{-1}=\pi\left(\epsilon_{\gamma} d_{e} L_{B}\right)^{1 / 2} /\left(d_{i} \beta^{1 / 2}\right)$, we obtain the dispersion relation

$$
\epsilon_{\gamma}=\frac{d_{i} \beta^{1 / 2} d_{e} \Delta^{\prime}}{\pi L_{B}}
$$

in the validity interval $\sigma \ll \tau \ll \sigma^{2}$ or

$$
\frac{d_{e}^{2} \Delta^{\prime 2}}{\pi^{2}} \gg \beta \gg \frac{d_{e}^{2} \Delta^{\prime 2} m_{e}}{\pi^{2} m_{i}},
$$


besides

$$
\epsilon_{\eta} \ll \frac{d_{i} \beta^{1 / 2} d_{e}^{3} k^{2} \Delta^{\prime}}{\pi L_{B}}
$$

The parametric region PR1 where $D(\sigma, \tau)=C$ and Hall effects are negligible can be reached only at yet lower values of beta. This way, we obtain the collisionless single-fluid dispersion relation

$$
\epsilon_{\gamma}=\frac{d_{e}^{3} \Delta^{\prime 2}}{C^{2} L_{B}}
$$

valid only for $\tau \gg \sigma^{2}$ or

$$
\beta \ll \frac{d_{e}^{2} \Delta^{\prime 2} m_{e}}{C^{2} m_{i}},
$$

besides

$$
\epsilon_{\eta} \ll \frac{d_{e}^{5} k^{2} \Delta^{\prime 2}}{C^{2} L_{B}}
$$

We point out that despite the stringent low limits on beta set in the above PR5 and PR1 collisionless regimes, those results are still compatible with our general requirement of subsonic growth rates, $\beta \gg \epsilon_{\gamma}^{2}$, which would be violated only with even lower betas.

In dimensionless reduced form, defining

$$
\tilde{\beta}=\frac{\beta C^{4}}{(1+\beta) \pi^{2} d_{e}^{2} \Delta^{\prime 2}} \quad \text { and } \quad \tilde{\gamma}=\frac{\epsilon_{\gamma} L_{B} C^{2}}{d_{e}^{3} \Delta^{\prime 2}}
$$

the growth rate of the collisionless tearing mode is

$$
\tilde{\gamma}(\tilde{\beta})= \begin{cases}1 & \text { for } \quad \tilde{\beta} \ll m_{e} / m_{i}(\mathrm{PR} 1) \\ \sqrt{\tilde{\beta} m_{i} / m_{e}} & \text { for } \quad m_{e} / m_{i} \ll \tilde{\beta} \ll 1(\mathrm{PR} 5) \\ \sqrt{m_{i} / m_{e}} & \text { for } 1 \ll \tilde{\beta}(\mathrm{PR} 3)\end{cases}
$$

and the functions $f_{6}$ and $f_{4}$ provide the smooth transitions through PR6 and PR4. 


\section{References}

[1] H. Furth, J. Killeen, and M. Rosenbluth, Physics of Fluids 6, 459 (1963).

[2] V. V. Mirnov, C. C. Hegna, and S. C. Prager, Physics of Plasmas 11, 4468 (2004).

[3] S. Bulanov, F. Pegoraro, and A. Sakharov, Physics of Fluids B 4, 2499 (1992).

[4] A. Fruchtman and H. Strauss, Physics of Fluids B 5, 1408 (1993).

[5] J. Drake and Y. Lee, Physics of Fluids 20, 1341 (1977).

[6] F. Pegoraro and T. Schep, Plasma Physics and Controlled Fusion 28, 647 (1986).

[7] B. Kuvshinov, Plasma Phys. Control. Fusion 36, 867 (1994).

[8] M. Abramowitz and I. Stegun, Handbook of Mathematical Functions, Dover, New York, 1965.

[9] R. Hazeltine and J. Meiss, Plasma confinement, Addison-Wesley, Redwood, CA, 1992. 


\begin{tabular}{lcc}
\hline & Alcator C-MOD & ITER \\
\hline$r(\mathrm{~m})$ & 0.2 & 2 \\
$B_{0}(\mathrm{~T})$ & 5 & 5 \\
$\epsilon_{B}$ & 0.33 & 0.33 \\
$n_{e}\left(10^{20} \mathrm{~m}^{-3}\right)$ & 2 & 1 \\
$T_{e}, T_{i}(\mathrm{keV})$ & 1 & 10 \\
$\eta\left(10^{-8} \Omega \mathrm{m}\right)$ & 4.7 & 0.18 \\
$d_{i}(\mathrm{~mm})$ & 23 & 32 \\
$d_{e}(\mathrm{~mm})$ & 0.38 & 0.53 \\
$\alpha\left(10^{-2}\right)$ & 34.3 & 4.8 \\
$\beta\left(10^{-2}\right)$ & 0.54 & 2.7 \\
$\epsilon_{\eta}\left(10^{-8}\right)$ & 10 & .027 \\
$\alpha \epsilon_{\eta}^{-1 / 5}$ & 8.5 & 4.0 \\
$\beta \epsilon_{\eta}^{-2 / 5}$ & 3.3 & 180 \\
$\gamma_{0}\left(\mathrm{~s}^{-1}\right)$ & 5200 & 21 \\
\hline
\end{tabular}

Table 1: Typical parameters in two representative tokamaks with deuterium ions. For reference, we use the characteristic single-fluid growth rate $\gamma_{0}=c_{A} k \epsilon_{\eta}^{3 / 5}$, with $k=3 / r$.

\begin{tabular}{l|cccccc}
\hline & PR1 & PR2 & PR3 & PR4 & PR5 & PR6 \\
\hline Mathematical & $\sigma \ll 1$ & $\sigma=O(1)$ & $\sigma \gg 1$ & $\sigma \gg 1$ & $\sigma \gg 1$ & $\sigma \gg 1$ \\
scaling & or & and & and & and & and & and \\
parameters & $\tau \gg \sigma^{2}$ & $\tau \ll 1$ & $\tau \ll \sigma$ & $\tau \sim \sigma$ & $\sigma \ll \tau \ll \sigma^{2}$ & $\tau \sim \sigma^{2}$ \\
\hline$B_{x}$-diffusion & $\bullet$ & $\bullet$ & $\bullet$ & $\bullet$ & $\bullet$ & $\bullet$ \\
$B_{z}$-diffusion & & $\bullet$ & $\bullet$ & $\bullet$ & & $\bullet$ \\
$B_{z}$-effects(non diffusive) & & & & & $\bullet$ & $\bullet$ \\
$\begin{array}{l}\text { Hall effects } \\
\text { Compressibility }\end{array}$ & & $\bullet$ & $\bullet$ & $\bullet$ & $\bullet$ & $\bullet$ \\
Two sublayers & & & & $\bullet$ & $\bullet$ & $\bullet$ \\
\hline $\begin{array}{l}\text { Primary } \\
\text { input } \\
\text { parameters }\end{array}$ & $\hat{\alpha} \ll 1$ & $\hat{\alpha} \sim 1$ & $\hat{\alpha} \gg 1$ & $\hat{\alpha} \gg 1$ & $\hat{\beta} \ll \hat{\alpha}^{-1 / 2}$ & $\hat{\alpha} \gg 1$ \\
\hline
\end{tabular}

Table 2: Main characteristics of parametric regions PR1 to PR6. A • means 'yes'.

\begin{tabular}{c|ccc}
\hline & PR1 & PR3 & PR5 \\
\hline$\epsilon_{\gamma} \sim$ & $\epsilon_{\eta}^{3 / 5} \epsilon_{B}^{2 / 5}(k L)^{-2}$ & $\epsilon_{\eta}^{1 / 2} \epsilon_{B}^{1 / 2}\left(k d_{i}\right)^{1 / 2}(k L)^{-5 / 2}$ & $\epsilon_{\eta}^{1 / 3} \epsilon_{B}^{2 / 3}\left(k d_{s}\right)^{2 / 3}(k L)^{-2}$ \\
$d_{2} / L \sim$ & $\epsilon_{\eta}^{2 / 5} \epsilon_{B}^{-2 / 5}(k L)^{-1}$ & $\epsilon_{\eta}^{1 / 4} \epsilon_{B}^{-1 / 4}\left(k d_{i}\right)^{3 / 4}(k L)^{-7 / 4}$ & $d_{s} / L$ \\
$d_{1} / L \sim$ & $\epsilon_{\eta}^{2 / 5} \epsilon_{B}^{-2 / 5}(k L)^{-1}$ & $\epsilon_{\eta}^{1 / 2} \epsilon_{B}^{-1 / 2}\left(k d_{i}\right)^{-1 / 2}(k L)^{-1 / 2}$ & $\epsilon_{\eta}^{2 / 3} \epsilon_{B}^{-2 / 3}\left(k d_{s}\right)^{-2 / 3}(k L)^{-1}$ \\
\hline
\end{tabular}

Table 3: Scaling laws for the growth rate and the width of the inner layers in different parametric regions, assuming $k^{-1} \Delta^{\prime} \simeq 2(k L)^{-2}$ (recall $k d_{i}=\alpha$ and $\left.k d_{s}=\alpha \beta^{1 / 2}\right)$. 

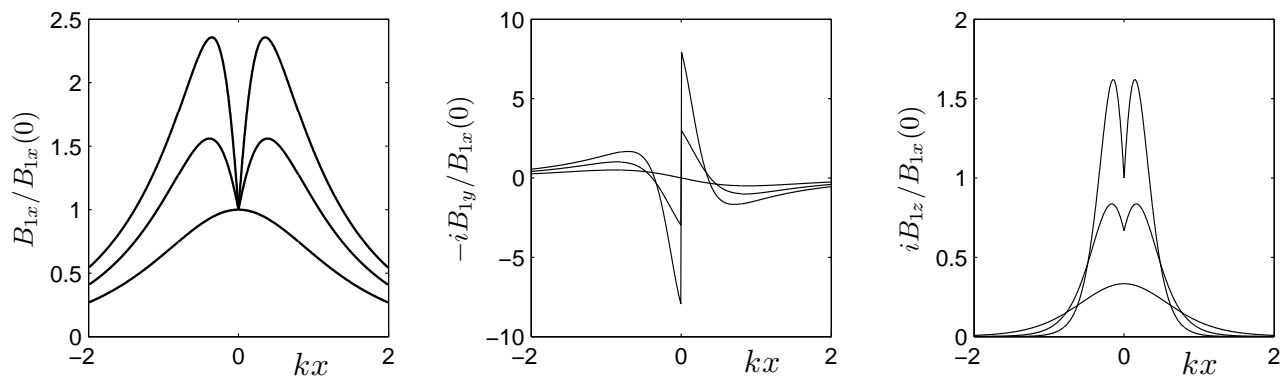

Figure 1: Components of the perturbed magnetic field $\boldsymbol{B}_{1}(x)$, for $\epsilon_{B}=1 / 3$ and sheet pinch profiles with $k L=1,1 / 2$, and $1 / 3$ (i.e. $\Delta^{\prime} \simeq 0,4$, and 13 ). 


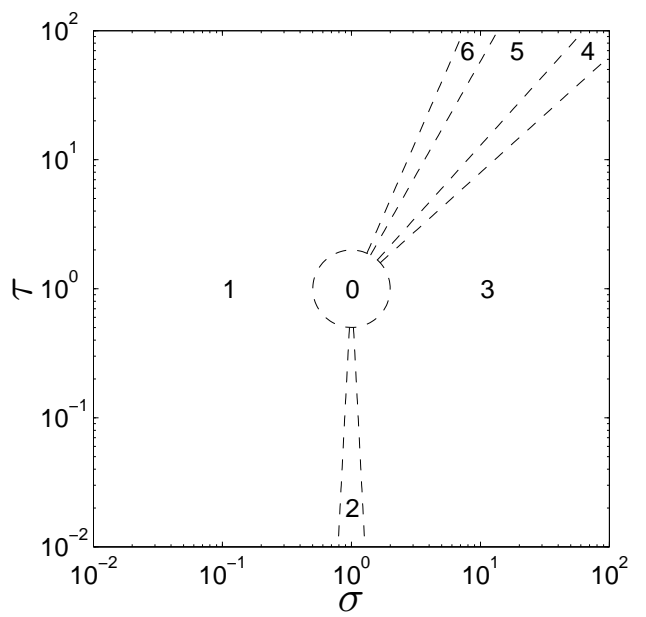

Figure 2: Location of the different asymptotic regions in the plane of mathematical scaling parameters. 

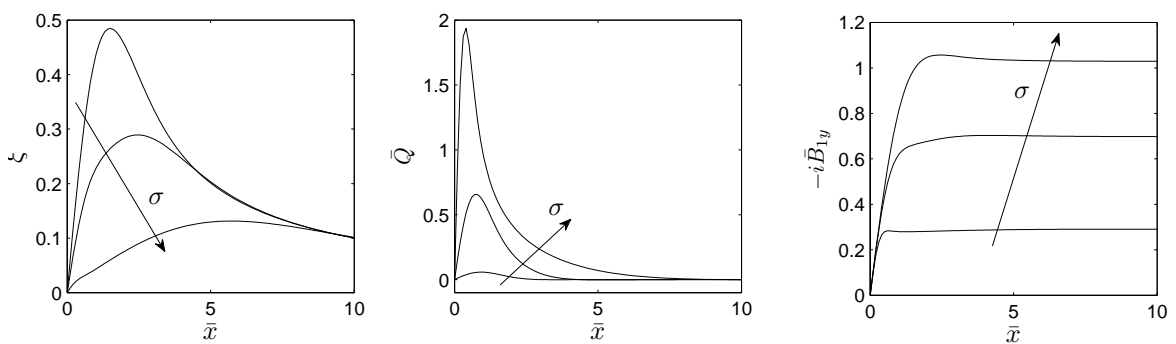

Figure 3: Inner solution for PR2 and $\sigma=0.6,3$, and 15. Notice that $\bar{x} \bar{Q}<0$ for $|\bar{x}| \gg 1$, Eq. (54). Here and in the next figure, $\bar{B}_{1 y}=-i B_{1 y} / B_{1 x}(0) \times \epsilon_{\eta} /\left(\epsilon_{\gamma} k d_{0}\right)$. 

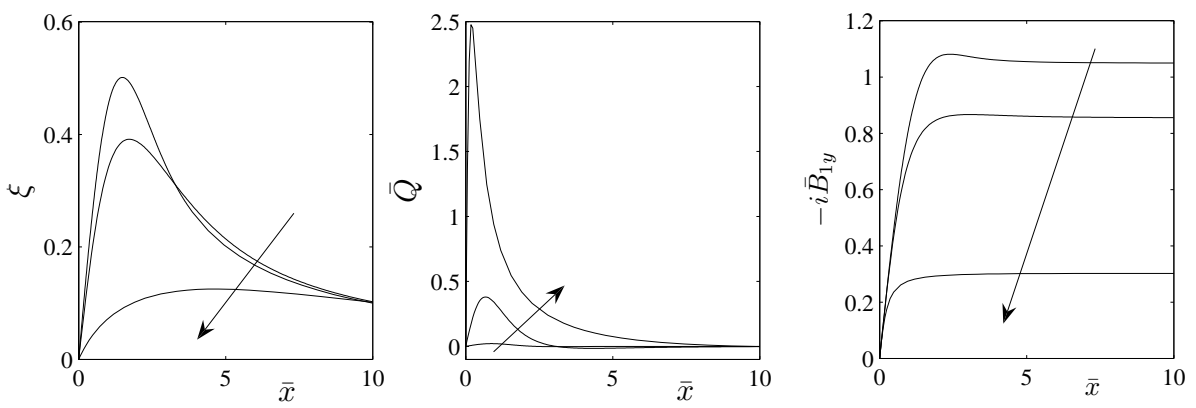

Figure 4: Inner solution for PR6 and $\sigma \tau^{-1 / 2}=0.2,1$, and 5 . 


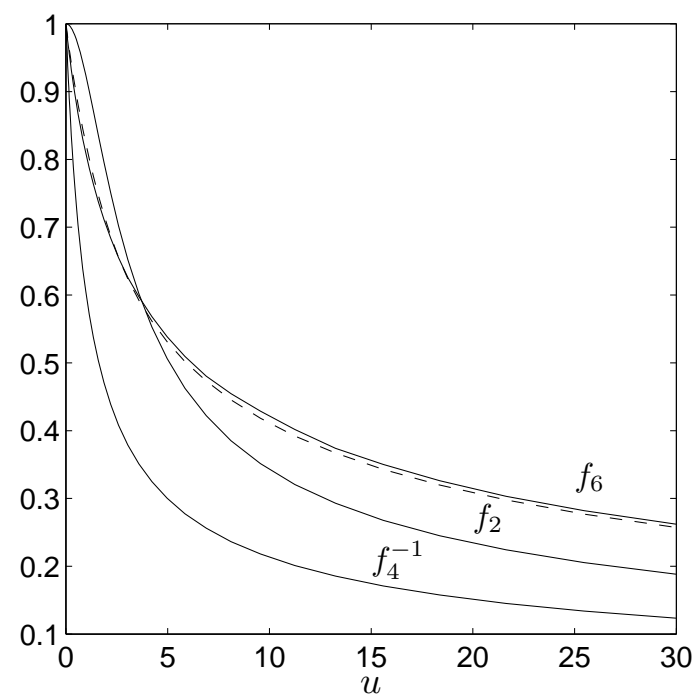

Figure 5: Functions $f_{2}(u), 1 / f_{4}(u)$, and $f_{6}(u)$. The dashed line is the approximate fit of $f_{6}$ in Eq. (92). 


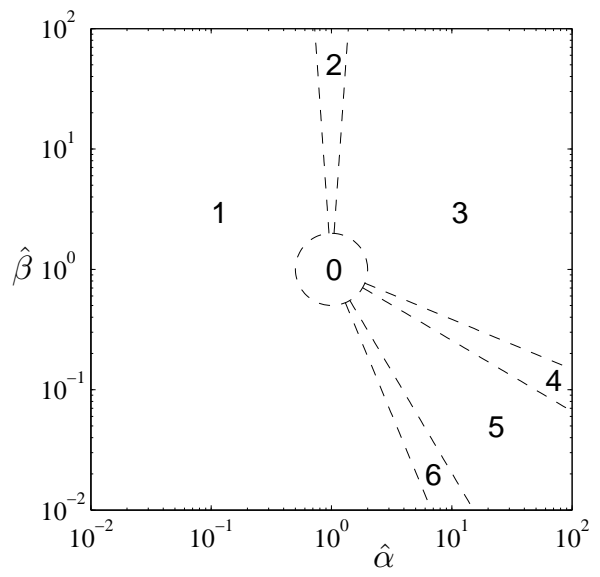

Figure 6: Location of the different asymptotic regions in the plane of primary input parameters. 


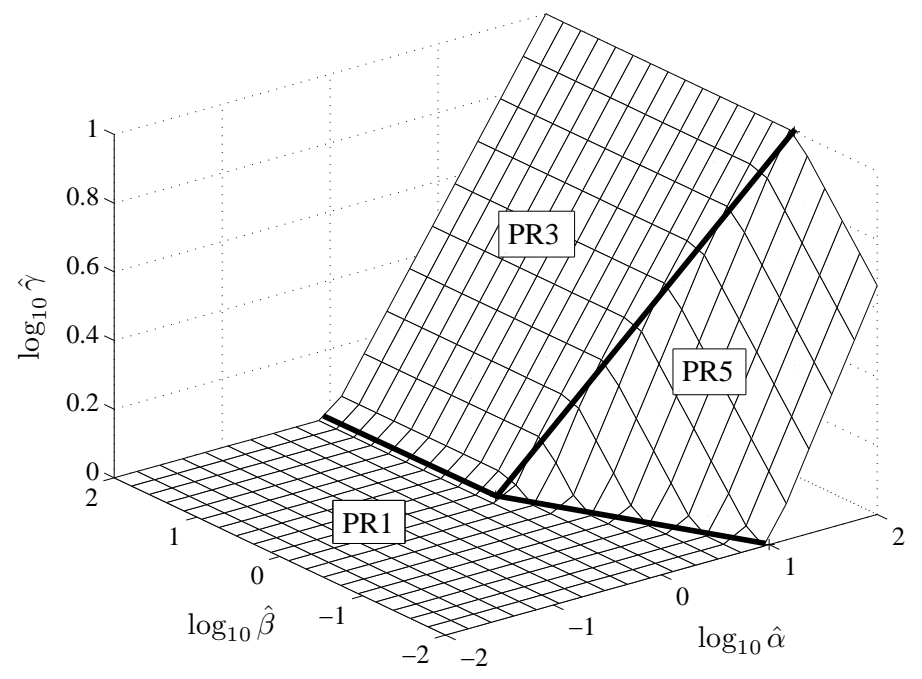

Figure 7: Growth-rate of the tearing mode, $\hat{\gamma}(\hat{\alpha}, \hat{\beta})$, in the different parametric regions. For illustration purposes, regions PR2, PR4, and PR6, have been reduced to lines and region PR0 to the intersection point. 


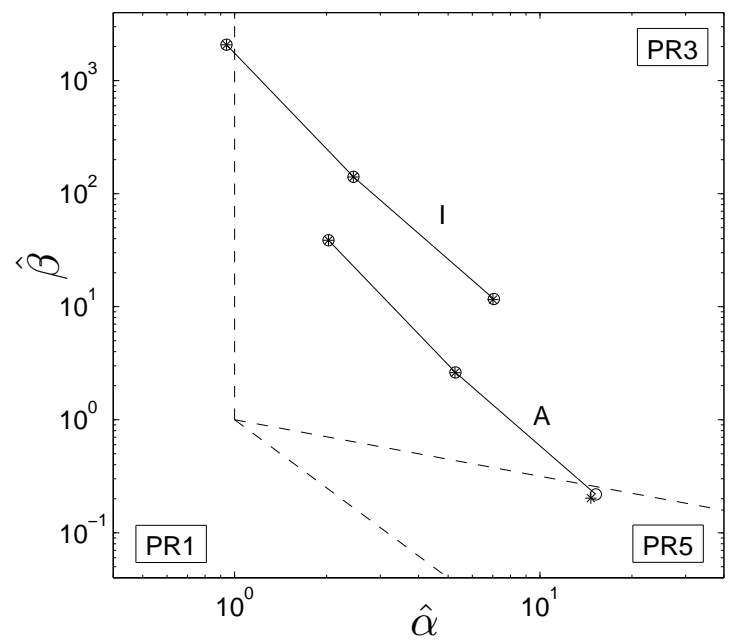

Figure 8: Parametric point locations for the Alcator C-MOD (A) and ITER (I) plasmas of Table 1 and three different values of $k^{-1} \Delta^{\prime}$. Points from left to right correspond to $k^{-1} \Delta^{\prime}=0.1,1$ and 10 , and circles and asterisks correspond, respectively, to $m_{e} / m_{i}=0$ and $1 / 3672$. The intermediate parametric regions PR2, PR4 and PR6 have been reduced to (dashed) lines in the $(\hat{\alpha}, \hat{\beta})$ plane, as in Figure 7. 